\title{
CHANGES IN REFRACTION IN DIABETES MELLITUS
}

\author{
BY
}

W. Stewart Duke-Elder, M.A., B.Sc., M.B., F.R.C.S. CLINICAL ASSISTANT, OPHTHALMOLOGICAL DEPARTMENT, ST. GEORGE'S HOSPITAL, LONDON

AMONG the rarer and more interesting of the ocular complications of diabetes mellitus are sudden changes in refraction. Both an increase and a decrease in the refractive power occur; myopia has long been recognised (occurring in 4 per cent. of cases, von Noorden ${ }^{(46)}$ ), hypermetropia is rarer. A considerable number of observers have reported cases of both conditions, and, on analysis, it would seem possible to co-ordinate their findings into a definite "law."-That the refractive power of the eye tends to vary directly as the sugar content of the blood; that is, there is a tendency to hypermetropia with decreased sugar, with increased sugar to myopia. This conclusion is strongly borne out by the study of three cases which have recently come under my observation in St. George's Hospital. In the first, refraction varied with mathematical precision with the blood-sugar content; the second showed hypermetropia following a decrease of sugar; while the third developed myopia as a terminal complication of a fatal diabetes.

\section{CASE I}

\section{Showing Refraction varying with Blood-Sugar Content}

W. J., male, aged 31 years. Previous health very good: led a healthy, active life, including four years service in France during the war. "For some years" had noticed his urine "muddy"; and in 1914 was turned down in an application for a life insurance policy. The case is, therefore, probably one of a mild symptomless diabetes of long standing, with, recently, an acute exacerbation. Unfortunately there was no record of his previous refraction, but he insisted that his sight.has always been perfect-in any case it allowed him to follow his occupation as a clerk with perfect ease.

Eight weeks before admission to hospital he noticed he was easily fatigued, and three days before he came under observation he collapsed entirely. On admission he appeared on the verge of coma: he was emaciated, shrunken, and anxious, his breath smelt strongly of acetone, and his urine was loaded with sugar.

After the institution of rigorous diabetic treatment with insulin he improved dramatically. But the treatment would appear to have been too heroic; his blood-sugar temporarily fell below normal, he developed symptons of hypoglycaemia, and, quite suddenly, on the following day, he complained of dimness and mistiness of vision. On examination he was found to have developed a pronounced hypermetropic astigmatism. 
The progress of the case can most easily be followed from the accompanying charts. The urine-sugar and acetone bodies (Fig. 1.) were estimated daily, the blood-sugar at somewhat longer intervals, and to co-ordinate the results several sugar tolerance tests were done, one of which is figured. (Fig. 4.) The nature of this curve incidentally proves that the patient was a true diabetic. The refractions were measured every third day, in each case under cycloplegia (Fig. 3.), and the appropriate lenses always brought his vision easily up to 6/5. During the whole period he was under observation the fundus and vessels remained normal, the media clear, the lens showed no trace of opacity, there was no spasm or paralysis of the external musculature, no esophoria, the visual fields remained unimpaired, and the tension normal.

Period A : $2 / 3 / 24$ to $12 / 3 / 24$.

The blood-sugar was rapidly reduced from an unknown height to 0.062 per cent. On $3 / 3 / 24$ the refraction read :

$$
\begin{aligned}
& \text { R.V.: + 5.25D. + cyl. } 0.75 \text { ax. } 150^{\circ} \text {. } \\
& \text { L.V.: + 4.75D. + cyl. } 0.75 \text { ax. } 30^{\circ} \text {. }
\end{aligned}
$$

Carbohydrates in the diet were increased and the insulin dosage lessened, and by 12/3/24 he was excreting 34.75 gms. of glucose per diem. During this period of sugar increase the refraction changed, as figured, to :

$$
\begin{aligned}
& \text { R.V.: + 2.0D. + cyl. } 0.75 \text { ax. } 150^{\circ} \text {. } \\
& \text { L.V.: + 3.0D. + cyl. } 0.25 \text { ax. } 30^{\circ} \text {. }
\end{aligned}
$$

Period B : $13 / 3 / 24$ to $24 / 3 / 24$.

The sugar again was rapidly reduced by insulin and starvation to an excretive value of $2.62 \mathrm{gms}$. per diem. After this second fall a second "hypermetropic crisis" occurred. On 14/3/24 the refraction gave :

$$
\begin{aligned}
& \text { R.V. }:+3.25 \text { D. + cyl. } 0.5 \text { ax. } 120^{\circ} \text {. } \\
& \text { L.V.: + 3.25D. + cyl. } 0.75 \text { ax. } 60^{\circ} \text {. }
\end{aligned}
$$

The blood-sugar was thereafter allowed to increase, latterly by largely adding to the carbohydrate intake and withholding insulin, until it reached 0.293 per cent. with a urine excretion of 169.05 gms. per diem, during which time a decrease in hypermetropia occurred. Refraction on $24 / 3 / 24$ gave -

$$
\begin{aligned}
& \text { R.V.: +1.25D. + cyl. } 0.25 \text { ax. } 120^{\circ} \text {. } \\
& \text { L.V.: + 1.25D. + cyl. } 0.25 \text { ax. } 60^{\circ} \text {. }
\end{aligned}
$$

Period C: $25 / 3 / 24$ to $25 / 4 / 24$.

A further reduction of sugar to 0.066 per cent. in the blood, and $1.55 \mathrm{gms}$. daily in the urine, caused hypermetropia again to appear : on $31 / 3 / 24$ :

$$
\begin{aligned}
& \text { R.V.: +2.0D. + cyl. } 1.25 \text { ax. } 105^{\circ} \\
& \text { L.V.: + 2.5D. + cyl. } 0.5 \text { ax. } 75^{\circ} \text {. }
\end{aligned}
$$


Thereafter his blood-sugar was kept approximately normal, i.e., at slightly less than 1 per cent., and the refraction fell as charted until it reached a value of :

$$
\begin{aligned}
& \text { R.V.: + 1.5D. + cyl. } 0.25 \text { ax. } 105^{\circ} \text {. } \\
& \text { L.V.: + 1.5D. + cyl. } 0.25 \text { ax. } 75^{\circ} \text {. }
\end{aligned}
$$

at which value it appeared to stabilize. By $24 / 4 / 24$ his metabolic condition appeared to have become stable on a fairly liberal diet aided by 30 units of insulin daily, his eyes appeared to have reached what was presumably their normal, and he himself felt, and looked fit and well. He was therefore discharged provided with instructions as to diet and insulin dosage.

Period D : $9 / 5 / 24$ to $19 / 5 / 24$.

Fourteen days later he returned and proceeded to go through a fourth sugar-variation phase, on this occasion pathological, when before it had been experimental. A badly sterilized insulin syringe at home had given him a cellulitis of the lef $\hat{\imath}$ arm. This was treated, but by $14 / 5 / 24$ the blood-sugar had risen to 0.292 per cent. and he was excreting $55.61 \mathrm{grms}$. in the urine per 24 hours. Meantime his refraction, which, when he came into hospital on the 9/5/24 was still at the previous (normal) value, tended towards myopia, and now read:

$$
\begin{aligned}
& \text { R.V.: + } 1.0 \text { D. + cyl. } 0.25 \text { ax. } 105^{\circ} \text {. } \\
& \text { L.V.: + } 0.75 \text { D. + cyl. } 0.5 \text { ax. } 75^{\circ} \text {. }
\end{aligned}
$$

Period E : $19 / 5 / 24$ to $9 / 7 / 24$.

The sugar remained high until 19/5/24, and the (comparative) myopia persisted. The arm then cleared up, the sugar fell to normal rapidly, and on the 24/5/24, when the blood-sugar value was 0.095 per cent., refraction showed a hypermetropia.

$$
\begin{aligned}
& \text { R.V.: + } 2.25 \text { D. + cyl. } 0.25 \text { ax. } 105^{\circ} \text {. } \\
& \text { L.V.: + } 2.0 \text { D. + cyl. } 0.5 \text { ax. } 75^{\circ}
\end{aligned}
$$

Thereafter he progressed steadily, and one week later was again discharged with a normal and stable blood-sugar, and a refraction once more reading :

$$
\begin{aligned}
& \text { R.V.: +1.5D. + cyl. } 0.25 \text { ax. } 105^{\circ} \text {. } \\
& \text { L.V.: +1.5D. + cyl. } 0.25 \text { ax. } 75^{\circ} \text {. }
\end{aligned}
$$

This persisted until July 19th.

\section{CASE II}

\section{Showing Hypermetropia with Sudden Decrease of Sugar}

L. L., female, aged 37 years. Previous health good ; diabetes had never been suspected. She gave a history of having been out-ofsorts and drowsy for one week, and of having vomited persistently 
for one day ; she had been without food of any kind for the previous forty-eight hours. On admission she was found to be in a state of semi-coma; she was very lethargic, her face was flushed, her breath smelt strongly of acetone, her urine was loaded with sugar and acetone-bodies, and her knee-jerks were absent. Her blood-sugar was found to be 0.493 per cent. Her eyes were in every respect perfectly normal, and her vision, as far as her state would permit of its being tested, was good. The refraction corresponded to that of glasses which were brought with her, obtained on prescription from an ophthalmic surgeon some two years previously, viz.:

$$
\begin{aligned}
& \text { R.V.: } 0 \text { D. + cyl. } 0.5 \text { ax. } 70^{\circ} \text {. } \\
& \text { L.V.: + } 0.5 \text { D. - cyl. } 1.5 \text { ax. } 160^{\circ} \text {. }
\end{aligned}
$$

Insulin in large doses was exhibited together with glucose by the mouth. Within the first twelve hours she had received 310 units, and on the following morning, with a blood-sugar value of 0.056 per cent. rising to 0.158 per cent., she was sitting up in bed comfortably eating breakfast. Her eyes were again examined in detail, and again found to be normal-fundus, media, lens, pupil reactions, accommodation, muscles, tension, etc. Unaided the V.A. gave :

$$
\begin{aligned}
& \text { R.V. }=6 / 5 . \\
& \text { L.V. }=6 / 9 .
\end{aligned}
$$

and, with her glasses, both eyes reached $6 / 5$.

Thereafter she was put on a diet of 1,500 calories (containing 74 gms. total glucose value) with a daily insulin dosage of 60 units falling gradually in 26 days to nil. Under this régime she remained perfectly well, her urine remained continuously sugar-free, and her blood-sugar at approximately the normal value of 0.1 per cent. The course of the case can be seen in figs. 5, 6 and 7 .

On the morning of the third day after admission her knee-jerks returned (an unusual event), and, in the afternoon, on awaking from a sleep, she burst into tears saying she had "gone blind." On examination her eyes were found to be normal (tension, pupils, fundi, movements, etc.), but the refraction showed a remarkable degree of hypermetropia. She was put under atropin, and investigation showed :

$$
\begin{aligned}
& \text { R.V.: +6.0D. + cyl. } 1.5 \text { ax. } 70^{\circ}=6 / 9 . \\
& \text { L.V.: +5.5D. + cyl. } 4.0 \text { ax. } 70^{\circ}=6 / 12 .
\end{aligned}
$$

-an addition of 9 dioptres in one axis.

She was kept atropinized during her stay in hospital, and her refraction steadily fell as charted in Fig. 7, until on $27 / 6 / 24$ it had reached a value of : 


$$
\begin{aligned}
& \text { R.V.: +1.0D. + cyl. } 1.0 \text { ax. } 70^{\circ}=6 / 5 . \\
& \text { L.V.: +2.0D. + cyl. } 2.0 \text { ax. } 70^{\circ}=6 / 6 \text {. }
\end{aligned}
$$

She was then discharged with instructions as to diet. On reporting on $9 / 7 / 24$ she was found to be still sugar-free, and her refraction, under cycloplegia, read :

$$
\begin{aligned}
& \text { R.V.: } \quad \text { 0D. }+ \text { cyl. } 1.0 \text { ax. } 70^{\circ}=6 / 5 . \\
& \text { L.V.: }+2.0 \text { D. }- \text { cyl. } 1.0 \text { ax. } 160^{\circ}=6 / 5 .
\end{aligned}
$$

On 19/7/24, seven weeks after admission, the refraction had almost reached its normal value :

$$
\begin{aligned}
& \text { R.V.: 0D. }+ \text { cyl. } 0.5 \text { ax. } 70^{\circ}=6 / 5 . \\
& \text { L.V.: }+1.0 \text { D. }- \text { cyl. } 1.0 \text { ax. } 100^{\circ}=6 / 5 .
\end{aligned}
$$

\section{CASE III}

\section{Showing Myopia with Increasing Sugar}

R.L., female, age 21 years. This patient was not directly under my care, but I had the privilege of observing her latterly. She was a diabetic of old standing, and had been treated for diabetes in hospital in June, 1923, when she was put on insulin treatment. She was re-admitted in coma in December of the same year, and again, also in coma, on April 4th, 1924.

The condition of her eyes on the two previous occasions was noted as normal, objectively and subjectively. She had never worn glasses, and her people insisted that her eyesight had always been perfect until a short time before the last admission, when she complained of dimness of vision, which steadily increased. I saw her two days after admission: she was extremely drowsy and irritable, her blood-sugar was 0.483 per cent., and her eyes, while in every other respect normal (including the tension), showed a refractive value under atropin of R. - 14 D. sph. L. - 13 D. sph. In spite of all treatment her condition got progressively worse. On the eighth day, while the refraction remained stationary, the tension of the eyeball had fallen to nil ; on palpation it felt soft like a water-bed. The next day the patient died.

Although in this case there is no record of any previous refraction, one would seem to be justified in assuming that there was some, probably a considerable, degree of terminal myopia.

These three cases thus show a gradual tendency to myopia with increasing sugar, and a definite hypermetropia, coming on suddenly, soon after a rapid decrease in the blood-sugar value.

A study of the literature has brought to light forty-five recorded cases illustrating this refractive change. These are tabulated below, the change in refraction, not the actual measurement, being given. 
Blood-sugar in Percentages.

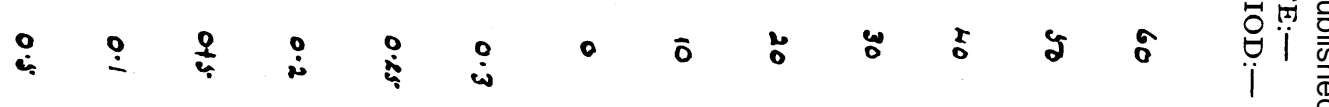
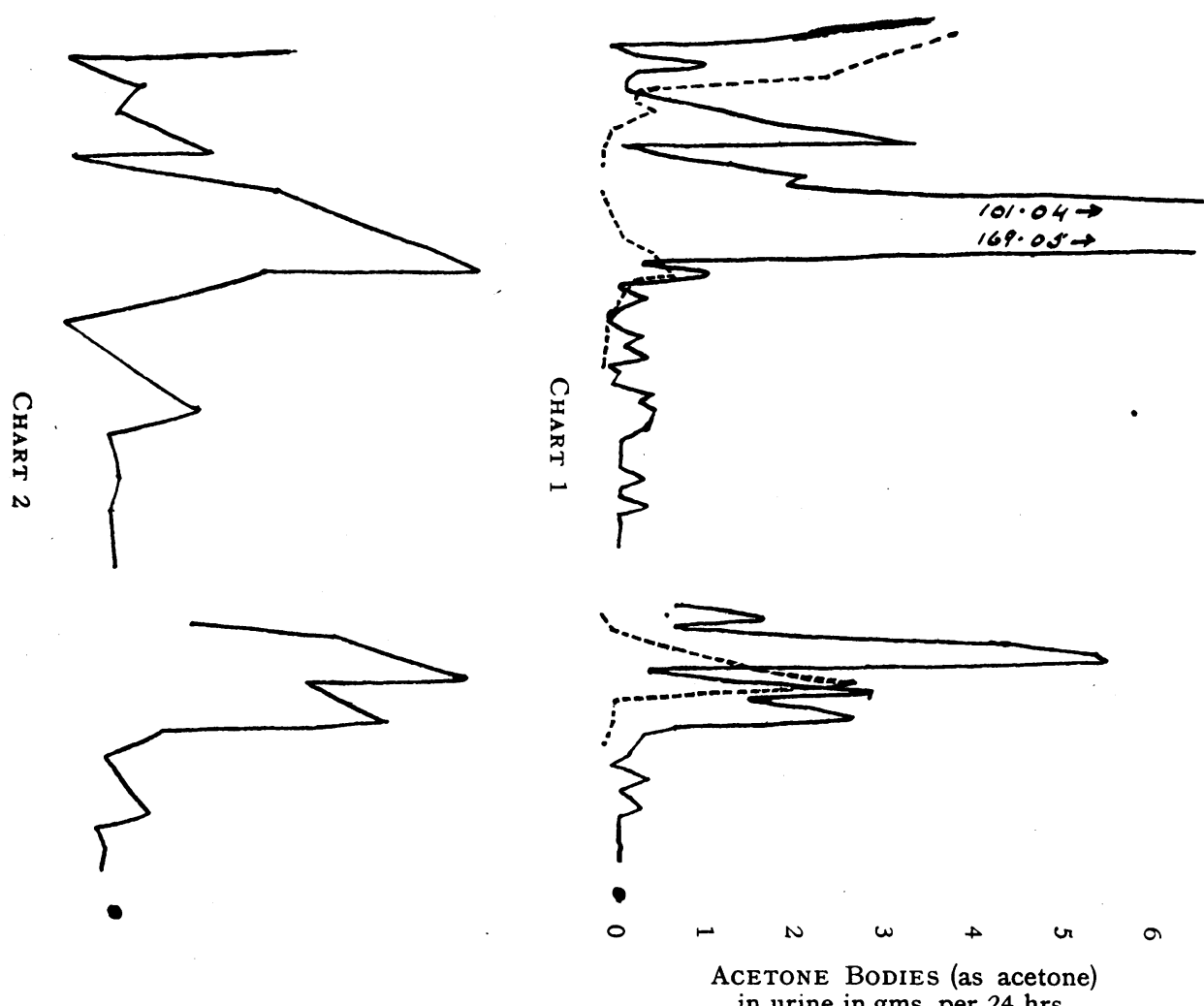

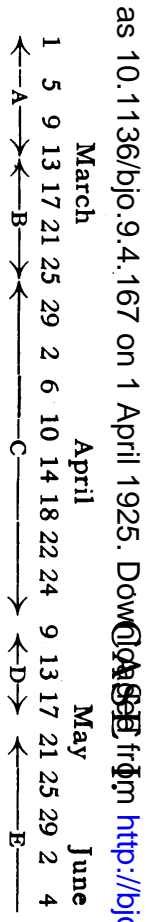

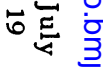

in urine in gms. per $24 \mathrm{hrs}$.

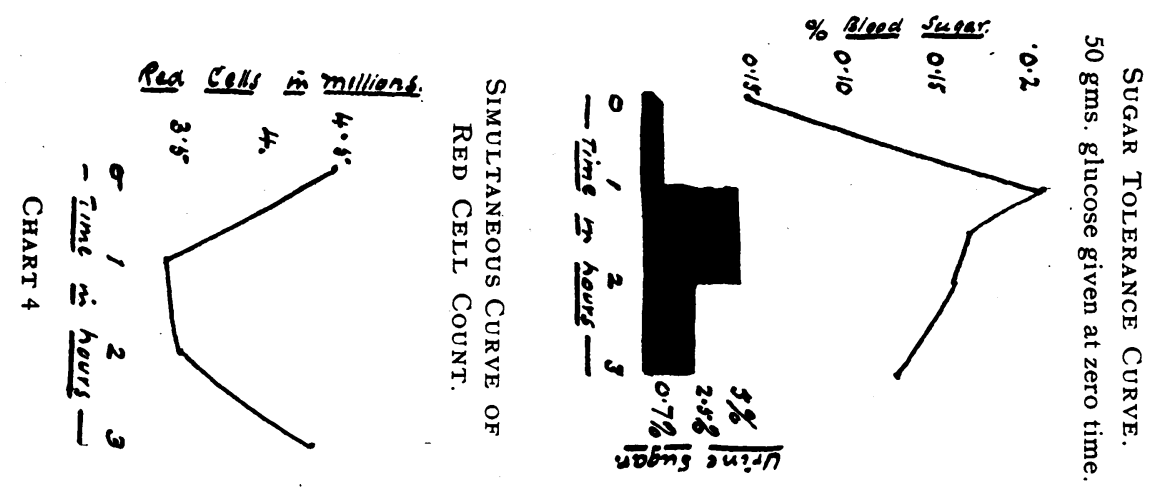


Changes in Refraction in Diabetes Mellitus 173
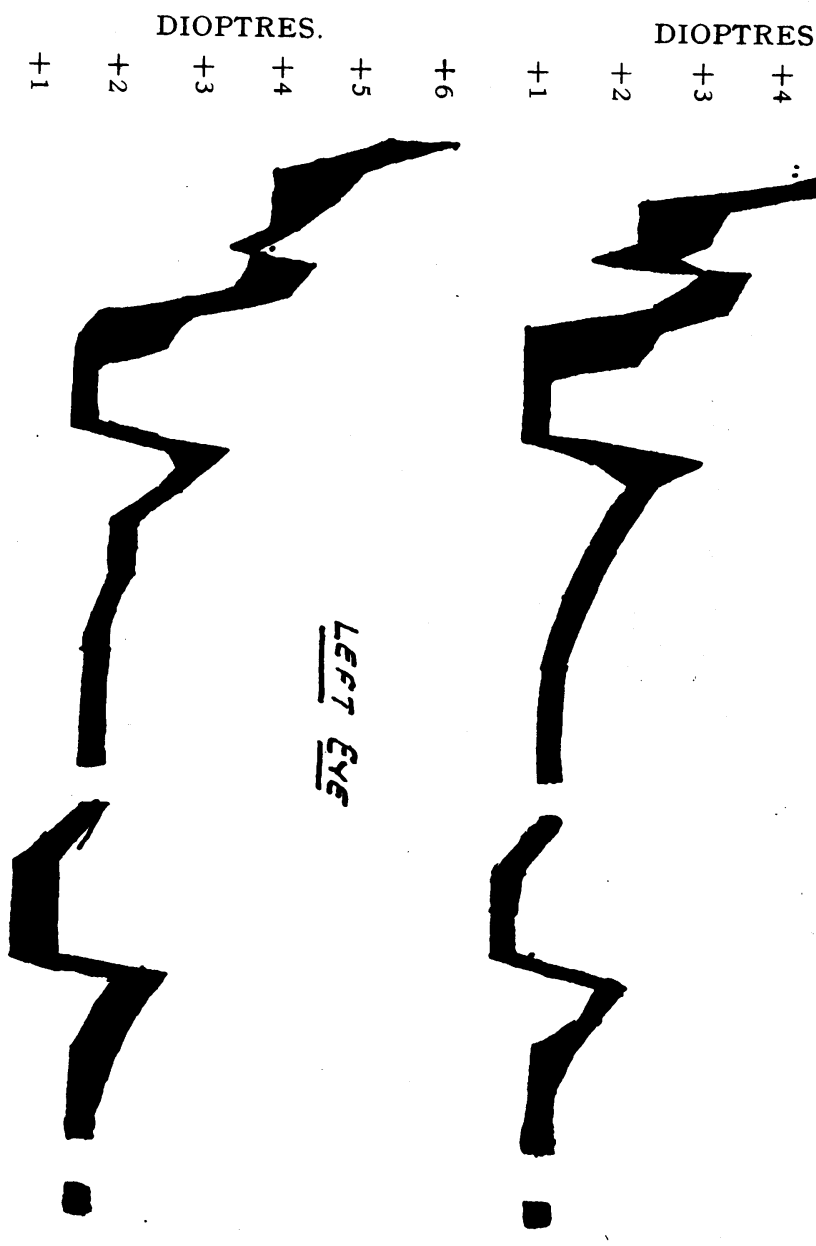

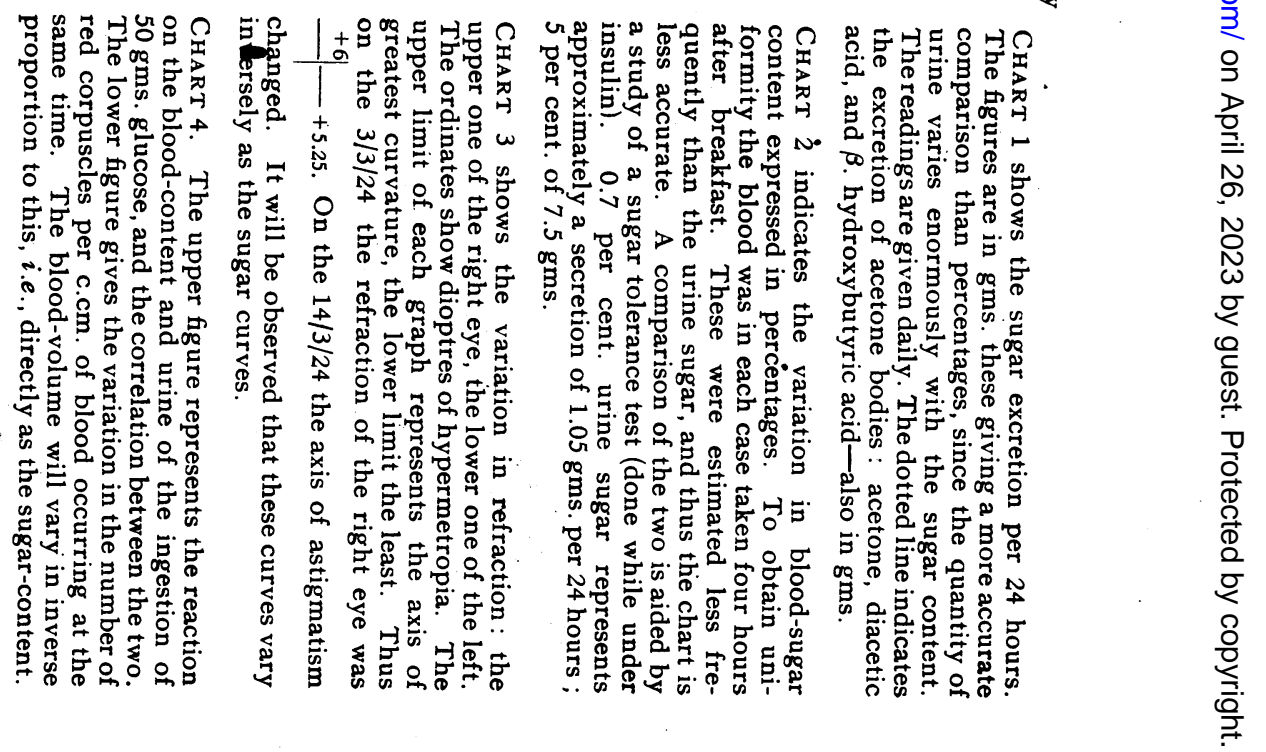


174 The British Journal of Ophthalmology

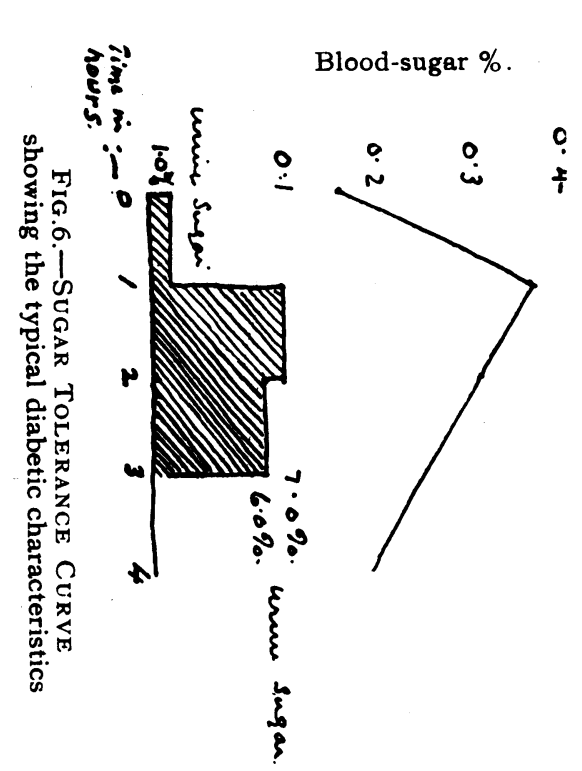

DIOPTRES.

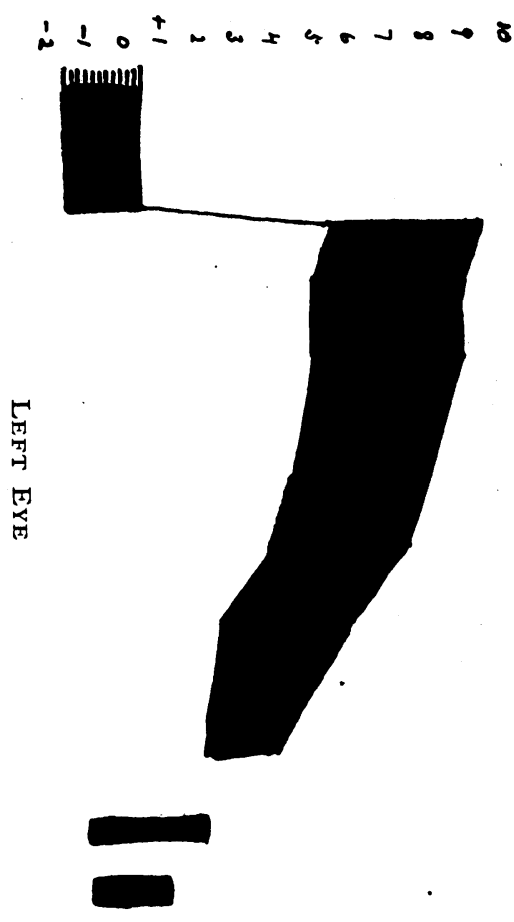

总

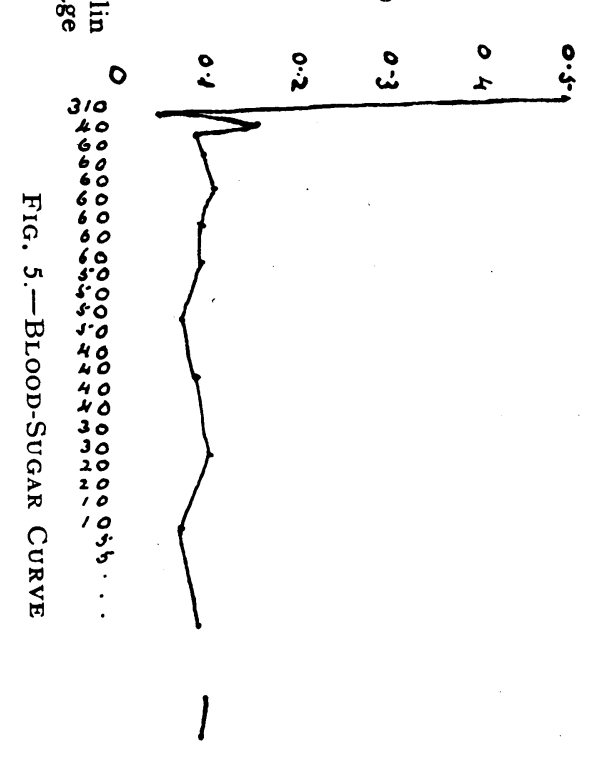

DIOPTRES.
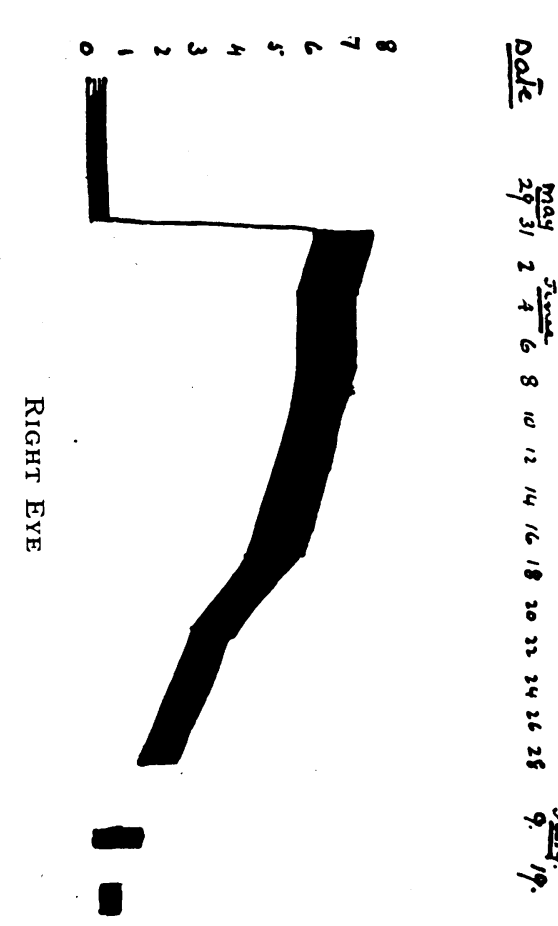

$\rightarrow$

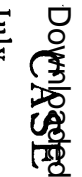

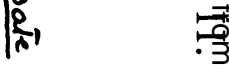

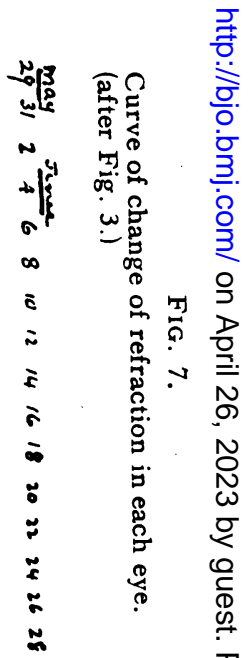

-

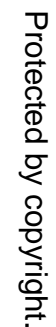


Changes in Refraction in Diabetes Mellitus

GROUP I

MYopia With SUgar INCREASE.

\begin{tabular}{|c|c|c|c|c|}
\hline No. & Observer. & $\begin{array}{c}\text { Sex and } \\
\text { Age. }\end{array}$ & $\begin{array}{l}\text { Change in } \\
\text { Refraction. }\end{array}$ & Clinical Notes. \\
\hline 1. & $\begin{array}{l}\text { Appenzeller, } \\
1896\end{array}$ & q 43. & -1 & $\begin{array}{l}\text { Returned to normal on } \\
\text { treatment. }\end{array}$ \\
\hline 2. & Grimsdale, 1897 & $\uparrow 45$. & $\begin{array}{c}\mathrm{R}:-3 \mathrm{D}-1.25 \text { cyl. } \\
\text { (axis changed) } \\
\mathrm{L}:-3.75 \mathrm{D} .\end{array}$ & $\begin{array}{l}\text { R. lens opacities centre. } \\
\text { Loss of accommodation. } \\
\text { Four weeks later coma and } \\
\text { death. }\end{array}$ \\
\hline 3. & $\begin{array}{l}\text { de Schweinitz, } \\
\text { No. } 1,1897 .\end{array}$ & 847 & -1.75 & - \\
\hline 4. & $\begin{array}{l}\text { de Schweinitz, } \\
\text { No. } 2,1897 .\end{array}$ & $\$ 20$. & $\begin{array}{l}R:-3 \\
L:-2 .\end{array}$ & $\begin{array}{l}\text { Oedema choroid : vitreous } \\
\text { opacities. Coma and death. }\end{array}$ \\
\hline 5. & Dujardin, 1900. & q 69. & -5 & $\begin{array}{l}\text { Sugar persisted on treat- } \\
\text { ment : eyes remained } \\
\text { myopic. }\end{array}$ \\
\hline 6. & Heine. & - & -1 & Cataract. \\
\hline 7. & $\begin{array}{l}\text { Neuberger, } \\
\text { No. } 1,1903 .\end{array}$ & $\$ 50$. & $\begin{array}{l}\mathrm{R}:-1.5 \\
\mathrm{~L}:-2 .\end{array}$ & $\begin{array}{l}\text { Returned to normal in three } \\
\text { weeks, al though sugar in- } \\
\text { creased. }\end{array}$ \\
\hline 8. & $\begin{array}{l}\text { Neuberger, } \\
\text { No. 2, } 1903 .\end{array}$ & \$ 48 . & $\begin{array}{l}R:-3.75 \\
L:-8.75\end{array}$ & Progressing cataract. \\
\hline 9. & Alexander, 1903. & 857. & -1 & $\begin{array}{l}\text { Urine Sugar, } 5.7 \text { per cent. } \\
\text { In } 13 \text { days sugar-free, and } \\
\text { refraction normal. }\end{array}$ \\
\hline 10. & Landolt, 1904. & $\$ 60 ?$ & ? & $\begin{array}{l}\text { Observed for several years. } \\
\text { Each exacerbation of dia- } \\
\text { betes announced itself by } \\
\text { a diminution of refraction. }\end{array}$ \\
\hline 11. & Jackson, 1905. & $\$ 52$. & -1.75 & Soon died. \\
\hline 12. & $\begin{array}{l}\text { Groenouw, } \\
1907 .\end{array}$ & $\$ 52$. & -1 & $\begin{array}{l}\text { Three weeks' treatment, } \\
\text { refraction normal. }\end{array}$ \\
\hline 13. & Wescott, 1911. & $\$ 47$. & $\begin{array}{c}\mathrm{R}:-1.5+0.25 \text { cyl. } \\
\mathrm{L}:-1.25+0.25 \text { cyl. } \\
.\end{array}$ & $\begin{array}{l}\text { Urine sugar } 5 \text { per cent. } \\
\text { Although sugar remained } \\
\text { high, refraction tended to } \\
\text { return to normal, adding } \\
+1.25 \mathrm{D} \text { of } \mathrm{Hm} \text {. in four } \\
\text { months. Coma and death } \\
\text { resulted. }\end{array}$ \\
\hline 14. & Elschnig. & 14 . 14 & -2.5 & $\begin{array}{l}\text { Urine sugar, } 7 \text { per cent. } \\
\text { t acetone. One month } \\
\text { later death in coma. }\end{array}$ \\
\hline
\end{tabular}




\section{GROUP II}

\section{Hypermetropia with Sugar Decrease}

\begin{tabular}{|c|c|c|c|c|}
\hline No. & Observer. & $\begin{array}{c}\text { Sex and } \\
\text { Age. }\end{array}$ & $\begin{array}{l}\text { Change in } \\
\text { Refraction. }\end{array}$ & Clinical Notes. \\
\hline 15. & $\begin{array}{l}\text { Hirschberg, } \\
1891 .\end{array}$ & б 48. & + sph. $1+$ cyl. 40 & $\begin{array}{l}\text { Patient myopic: normal } \\
-9 D \text {. sph. Change occurred } \\
\text { 14th day of treatment. }\end{array}$ \\
\hline 16. & $\begin{array}{l}\text { Risley, No. 3, } \\
\text { 1897. }\end{array}$ & $?$ & +1.25 cyl. ax. $90^{\circ}$ & $\begin{array}{l}\text { Appeared and disappeared } \\
\text { with paresis of accommod- } \\
\text { ation and esophoria. Due } \\
\text { to change in corneal } \\
\text { curvature. }\end{array}$ \\
\hline 17. & Gallus, 1906. & 851. & $\begin{array}{l}\mathrm{R}:+1.5 \\
\mathrm{~L}:+2 .\end{array}$ & $\begin{array}{l}\text { Occurred on second week of } \\
\text { treatment. Normal in two } \\
\text { months, when sugar-free. }\end{array}$ \\
\hline 18. & $\begin{array}{l}\text { Lundsgaard, } \\
\text { No. 1, } 1907 .\end{array}$ & १? & $\begin{array}{l}\mathrm{R}:+2 . \\
\mathrm{L}:+1.5\end{array}$ & $\begin{array}{l}\text { Occurred suddenly on treat- } \\
\text { ment. Normal in four } \\
\text { months when sugar-free. }\end{array}$ \\
\hline 19. & $\begin{array}{l}\text { Lundsgaard, } \\
\text { No. } 2,1910 .\end{array}$ & \$ 50 . & $\begin{array}{l}\mathrm{R}:+1.5 \\
\mathrm{~L}:+3 .\end{array}$ & $\begin{array}{l}\text { Occurred } 15 \text { days after } \\
\text { treatment } \\
\text { During first } 12 \text { days urine } \\
\text { sugar reduced from } 6.2 \text { per } \\
\text { cent. to } 0.2 \text { per cent. }\end{array}$ \\
\hline 20. & $\begin{array}{l}\text { Knapp, No. 2, } \\
1909 .\end{array}$ & के 48. & +1 & $\begin{array}{l}\text { Paralysis of accommodation. } \\
\text { Occurred a few weeks } \\
\text { after treatment began. } \\
\text { Normal in four weeks } \\
\text { when sugar-free. }\end{array}$ \\
\hline 21 & $\begin{array}{l}\text { Ellis, No. } 1, \\
\text { 1911. }\end{array}$ & $\$ 40$. & +4.5 & $\begin{array}{l}\text { Occurred two to three } \\
\text { weeks after treatment be- } \\
\text { gan. Returned towards } \\
\text { normal slowly with the } \\
\text { appearance of a small cy- } \\
\text { linder. In three months } \\
\text { when sugar was still } \\
2.5 \text { per cent. } \mathrm{R}:+2.25 \\
\mathrm{~L}:+1.37 \text {. }\end{array}$ \\
\hline 22. & Ellis, No. 4. & $?$ & +1.5 D. + 0.5 cyl. & $\begin{array}{l}\text { Occurred on treatment one } \\
\text { week. }\end{array}$ \\
\hline
\end{tabular}


Changes in Refraction in Diabetes Mellitus

GROUP II.-continued.

Hypermetropia with Sugar Decrease

\begin{tabular}{|c|c|c|c|c|}
\hline No. & Observer. & $\begin{array}{l}\text { Sex and } \\
\text { Age. }\end{array}$ & $\begin{array}{l}\text { Change in } \\
\text { Refraction. }\end{array}$ & Clinical Notes. \\
\hline 23. & $\begin{array}{l}\text { Zentmayer, } \\
1912 .\end{array}$ & 58 . & $\begin{array}{l}\mathrm{R}:+2.5+0.25 \\
\mathrm{~L}:+2.5+0.5\end{array}$ & $\begin{array}{l}\text { Occurred } 10 \text { days after } \\
\text { treatment started. Sugar } \\
\text { reduced from } 5 \text { per cent. to } \\
\text { nil. In two months re } \\
\text { fraction almost normal } \\
R: 0.5 \mathrm{D} \text {. L : } 0.75 \mathrm{D} \text {. }\end{array}$ \\
\hline 24. & Post, 1917. & \&5. & $\begin{array}{l}\mathrm{R}:+0.75 \\
\mathrm{~L}:+1\end{array}$ & $\begin{array}{l}\text { ? on treatment. Normal in } \\
\text { four months. }\end{array}$ \\
\hline 25. & Spalding, 1923. & \$29. & $\begin{array}{l}\mathrm{R}: 3.75+0.75 \text { cyl. } \\
\mathrm{L}: 3.5+0.25 \text { cyl. }\end{array}$ & $\begin{array}{l}\text { Occurred on second day's } \\
\text { starvation. }\end{array}$ \\
\hline 26. & $\begin{array}{l}\text { Roberts, No. } 1 \text {, } \\
1923 .\end{array}$ & 851 . & +1.75 & $\begin{array}{l}\text { Occurred two weeks after } \\
\text { - treatment began. Sugar } \\
\text { reduced from } 7 \text { per cent. to } \\
\text { nil in four weeks. Normal } \\
\text { in six months. }\end{array}$ \\
\hline 27. & $\begin{array}{l}\text { Roberts, No. } 2 \text {, } \\
\text { 1923. }\end{array}$ & \& 54 . & $\begin{array}{l}\mathrm{R}: \mathrm{Nil} \\
\mathrm{L}:+1.75\end{array}$ & $\begin{array}{l}\text { Occurred after one week's } \\
\text { dieting. Five weeks later } \\
\text { returned to normal with a } \\
\text { fall of } 1.25 \mathrm{D}+0.25 \text { cyl. } \\
\text { in R.E. }\end{array}$ \\
\hline 28. & $\begin{array}{l}\text { Roberts, No. } 4 \text {, } \\
1923 .\end{array}$ & 55 . & $+4+0.25$ cyl. & $\begin{array}{l}\text { Occurred on sugar falling } \\
\text { from } 10 \text { per cent. to nil. } \\
\text { Normal in three months. } \\
\text { Later, on restricted diet, } \\
\text { vision blurs ; on eating } \\
\text { carbohydrate, vision clears } \\
\text { up. }\end{array}$ \\
\hline 29. & $\begin{array}{l}\text { Roberts; No. } 5 \text {, } \\
1923 .\end{array}$ & $\$ 66$. & $\begin{array}{l}\mathrm{R}:+3.25 \\
\mathrm{~L}:+2.75\end{array}$ & $\begin{array}{l}\text { On treatment four weeks. } \\
\text { Sugar reduced from } 7 \text { per } \\
\text { cent. Normal in three } \\
\text { weeks. }\end{array}$ \\
\hline 30. & $\begin{array}{l}\text { Elschnig, No. } 1 \text {, } \\
1923 .\end{array}$ & ९ 47. & $\begin{array}{l}\mathrm{R}:+2 . \\
\mathrm{L}: \text { no change. }\end{array}$ & $\begin{array}{l}\text { Occurred } 14 \text { days after } \\
\text { treatment. Three months } \\
\text { later was returning to nor- } \\
\text { mal. The L.E. had had a } \\
\text { previous cataract extract- } \\
\text { ion. It remained constant } \\
+9.5 \text { D sph. }+4 \text { cyl. }= \\
6 / 12 \text {. }\end{array}$ \\
\hline
\end{tabular}




\section{GROUP III}

\section{SHOWING BOTH CHANGES}

\begin{tabular}{|c|c|c|c|c|}
\hline No. & Observer. & $\begin{array}{c}\text { Sex and } \\
\text { Age. }\end{array}$ & $\begin{array}{l}\text { Change in } \\
\text { Refraction. }\end{array}$ & Clinical Notes. \\
\hline 31. & $\begin{array}{l}\text { Risley, No. } 1 \text {, } \\
\text { 1897. }\end{array}$ & 49 . & $\begin{array}{l}\text { (a) } \mathrm{R}:+2.25+1.25 \\
\mathrm{~L}:+3.25+0.75 \\
\text { cyl. } \\
\text { cyl. } \\
\text { (b) } \mathrm{R}:-3.75 . \\
\mathrm{L}:-4.5 .\end{array}$ & $\begin{array}{l}\text { (a) Occurred on commence- } \\
\text { ment of treatment: i.e., } \\
\text { sugar diminished. } \\
\text { (b) } 25 \text { days later: sugar in- } \\
\text { creased. }\end{array}$ \\
\hline 32. & Risley, No. 2 & б 74. & $\begin{array}{l}\text { (a) } \mathrm{R}:+1 . \mathrm{L}:+0.75 \\
\text { (b) } \mathrm{R}:-1 . \mathrm{L}:+0.75\end{array}$ & $\begin{array}{l}\text { (a) On treatment } 14 \text { days: } \\
\text { sugar a trace. } \\
\text { (b) One month later : sugar } \\
4.2 \text { per cent. }\end{array}$ \\
\hline 33. & Carpenter, 1902. & 51 . & $\begin{array}{r}\text { (a) } \mathrm{R}:+1.75+0.5 \\
\text { cyl. } \\
\mathrm{L}:+2.5+0.5 \\
\text { cyl. } \\
\text { (b) } \mathrm{R}:-2.25 \mathrm{~L}:-3 .\end{array}$ & $\begin{array}{l}\text { (a) On treatment four weeks. } \\
\text { (b) Patient left off diet. }\end{array}$ \\
\hline 34. & Gould, 1907. & 858. & $\begin{array}{r}\text { (a) } \mathrm{R}:-0.63 \mathrm{D} . \\
\mathrm{L}:-0.63 \mathrm{D} .-0.25 \\
\text { cyl. (axis changed) } \\
\text { (b) } \mathrm{R}:+1.25+0.13 \\
\mathrm{cyl} \\
\mathrm{L}:+1.38+0.13 \\
\text { cyl. (axis changed). }\end{array}$ & $\begin{array}{l}\text { (a) Untreated. } \\
\text { (b) On treatment. }\end{array}$ \\
\hline 35. & $\begin{array}{l}\text { Knapp, No. } 1 \text {, } \\
\text { 1909. }\end{array}$ & † 47 . & $\begin{array}{l}\text { (a) }-2 . \\
\text { (b) } \mathrm{R}:+3 . \mathrm{L}:+2.5 \\
\text { (c) } \mathrm{R}:-0.5 . \mathrm{L}:-0.25\end{array}$ & $\begin{array}{l}\text { (a) Untreated. } \\
\text { (b) After } 14 \text { days' treatment } \\
\text { when sugar-free. } \\
\text { (c) After } 14 \text { days' sugar-free } \\
\text { (returning to normal). }\end{array}$ \\
\hline 36. & $\begin{array}{l}\text { Lundsgaard, } \\
\text { No. } 3,1910 \text {. }\end{array}$ & $\subsetneq 50$. & $\begin{array}{l}\text { (a) } \mathrm{R}:+1 . \mathrm{L}:+0.5 \\
\text { (b) } \mathrm{R}:-2.5 . \mathrm{L}:-2 .\end{array}$ & $\begin{array}{l}\text { (a) Sugar reduced from } 6 \\
\text { per cent. to } 0.85 \text { per cent. } \\
\text { (b) Sugar increasing. }\end{array}$ \\
\hline 37. & $\begin{array}{l}\text { Lundsgaard, } \\
\text { No. 4, } 1919 .\end{array}$ & 841. & $\begin{array}{l}\text { (a) }+2.5 \\
\text { (b) }-2 . \\
\text { (c) }-0.5\end{array}$ & $\begin{array}{l}\text { (a) Ten days' treatment: } \\
\text { sugar, urine nil ; blood } \\
0.120 \text { per cent. } \\
\text { (b) Six days later : sugar, } \\
\text { urine } 2.4 \text { per cent. ; blood } \\
0.217 \text { per cent. } \\
\text { (c) Six days later : sugar, } \\
\text { urine } 0.3 \text { per cent. ; blood } \\
0.180 \text { per cent. }\end{array}$ \\
\hline 38. & $\begin{array}{l}\text { Ellis, No. } 3 \text {, } \\
\text { 1911. }\end{array}$ & $\uparrow 65$. & $\begin{array}{r}\text { (a) } \mathrm{R}:+2.5+1.25 \\
\text { cyl. } \\
\mathrm{L}:+2.5+0.75 \\
\text { cyl. (axis changed). } \\
\text { (b) } \mathrm{R}:-1.75-1.0 \\
\mathrm{~L}:-1.75-0.75 \\
\text { cyl. (axis changed). }\end{array}$ & $\begin{array}{l}\text { (a) On treatment, four } \\
\text { weeks. } \\
\text { (b) Five months later, } \\
\text { treatment neglected. }\end{array}$ \\
\hline
\end{tabular}


Changes in Refraction in Diabetes Mellitus

GROUP IV

Anomalous Cases, and Cases lacking Sugar Data

\begin{tabular}{|c|c|c|c|c|}
\hline No. & Observer. & $\begin{array}{l}\text { Sex and } \\
\text { Age. }\end{array}$ & $\begin{array}{l}\text { Change in } \\
\text { Refraction. }\end{array}$ & Clinical Notes. \\
\hline 39. & Horner, 1873. & 55. & $\begin{array}{l}\text { (a) }+2 \text {. } \\
\text { (b) }-2 \text {. }\end{array}$ & $\begin{array}{l}\text { No mydriatic used. } \\
\text { No sugar data. }\end{array}$ \\
\hline 40. & Cohen, 1878. & $q 68$. & +1.5 & $\begin{array}{l}\text { No mydriatic used. } \\
\text { No sugar data. }\end{array}$ \\
\hline 41. & Doyne, 1897. & 840. & $\begin{array}{l}\text { (a) }+3 . \\
\text { (b) }-2.25 \text {. }\end{array}$ & $\begin{array}{l}\text { Was }+3 \mathrm{D} \text {. when had sugar } \\
+ \text { was } 0.75 \mathrm{D} \text {. when sugar- } \\
\text { free some months later } \\
\text { but no data of condition } \\
\text { before or during the in- } \\
\text { terval. }\end{array}$ \\
\hline 42. & Sourdille, 1900. & 53. & $+2 \mathrm{D}$ & $\begin{array}{l}\text { Developed suddenly : nor- } \\
\text { mal in eight days when } \\
\text { sugar-free. No mydriatic } \\
\text { used. Syphilis }+ \text {. No } \\
\text { definite sugar data. }\end{array}$ \\
\hline 43. & $\begin{array}{l}\text { Lichtenstein, } \\
1906 .\end{array}$ & 817 & +2 & $\begin{array}{l}\text { Apparently a Hm. with } \\
\text { sugar }+ \text {. It lasted } 14 \text { days, } \\
\text { and returned to normal } \\
\text { some weeks later. Ac- } \\
\text { commodation was para- } \\
\text { lysed, adding }+13 \mathrm{D} \text {. (sic) } \\
\text { for near vision. No definite } \\
\text { sugar data. }\end{array}$ \\
\hline 44. & $\begin{array}{c}\text { Ellis, No. 2, } \\
1911 .\end{array}$ & \& 48. & $\begin{aligned} \text { (a) } \mathrm{R}:-0.25 \text { cyl. } \\
\text { (axis changed) } \\
\mathrm{L}:-0.25 \mathrm{D} . \\
\text { (b) } \mathrm{R}:-1.75 . \\
\mathrm{L}:-2.0 . \\
\text { (c) } \mathrm{R}:+0.25 . \\
\mathrm{L}:+0.5+0.25 \text { cyl. }\end{aligned}$ & $\begin{array}{l}\text { (a) Measured when urine } \\
\text { sugar was } 4.5 \text { per cent. } \\
\text { (b) Three months later, } \\
\text { when sugar-free, i.e., an } \\
\text { apparent myopia with } \\
\text { sugar decrease; but esti- } \\
\text { mations only at prolonged } \\
\text { intervals. } \\
\text { (c) Two months later. }\end{array}$ \\
\hline 45. & $\begin{array}{l}\text { Roberts, No. 3; } \\
1923 .\end{array}$ & $\$ 53$. & $+1.5+0.25$ cyl. & $\begin{array}{l}\text { Normal in four months. } \\
\text { Left chorio-retinitis. Right } \\
\text { swollen disc. Patient al- } \\
\text { most blind. No definite } \\
\text { sugar data. }\end{array}$ \\
\hline
\end{tabular}

To account for this refractive change a great number of theories have at various times been advanced, including the suggestion that refractive errors are a causal factor in the aetiology of diabetes. The possible causes may be considered briefly : most can as briefly be dismissed. 


\section{DYNAMIC.-Dependent on a neuro-muscular mechanism}

1. Affecting the internal musculature.

Paresis of accommodation, allowing a latent hypermetropia to become manifest, in part or in whole (Groenouw, SchmidtRimpler $\left.{ }^{(12)}\right)$.

This does occur in some cases (Nos. 16, 20,43); it may account for part or the whole of the change in some (Nos. 39, 40,42); but, applied to the majority, the theory breaks down.

Thus: (a) The range of accommodation has repeatedly been found to be normal.

(b) A mydriatic eliminates its influence.

(c) The ages of many of the cases are beyond that during which accommodation is active (Nos. 5, 29, $38,40)$.

(d) Many of the cases developed a degree of hypermetropia greater than the range of accommodation would allow (Nos. 5, 8).

(e) It cannot account at all for the occurrence of myopia.

2. Affecting the external musculature (Risley ${ }^{(16)}$ ).

In Case 16, the hypermetropic astigmatism, apparently due to a change in corneal curvature, which appeared and disappeared with esophoria, presumably depended on a spasm of the laterally acting muscles. But this would appear to be isolated, and, when it has been looked for specially in several cases, it has been found absent.

\section{STATIC.-Dependent on a physico-chemical mechanism}

1. Affecting the coats of the eye.

(i) A shortening of the antero-posterior axis due to a shrinkage of the eye from loss of fluid in the vitreous. (Sourdille ${ }^{(42)}$ : Lichtenstein ${ }^{(43)}$.)

This undoubtedly does occur in advanced cases, as a terminal event in diabetes when the tissues generally have become dehydrated. It is well known clinically as the "soft eye" of diabetic coma.

But (a) If it occured at all in milder cases the result would be a hypermetropia with increasing sugar. The theory was advanced to explain the anomalous cases already dealt with.

(b) The tension has been repeatedly measured and found to be normal.

(ii) A lengthening of the antero-posterior axis, due to softening and yielding of the sclera, the underlying pathology being a diffuse oedematous condition of the choroid. (de Schweinitz and Kneis ${ }^{(4)}$.) 
But (a) although this might have occurred in one case (No. 4), when the diabetes was very severe, and the eyes otherwise gravely affected, in milder cases the theory is not tenable, and clinically no such choroiditis occurs.

(b) Such a theory cannot account for an initial hypermetropia.

2. Affecting the media generally-an index change. A general alteration in the index of refraction due to the presence of sugar and other metabolites, rendering the refraction greater, and thus inducing myopia as diabetes progressed, rendering it less, and inducing hypermetropia with a decrease in the severity of the disease. (Landolt and de Wecker ${ }^{(48)}$.)

This seems delightfully simple, but :

(a) The blood-volume normally contains a very small actual amount of sugar. Recent work ${ }^{(49)}$ has placed the blood-volume at $1 / 13$ of the body weight. With an average blood-sugar value of 0.1 per cent., an average man of 65 kilos. will have normally circulating in the blood 5 gms. of sugar. The highest diabetic value found in a very extensive investigation ${ }^{(50)}$ was 15 gms. of total sugar, or 10.75 gms. of plasma sugar. The increase, therefore, at any time is small, in the eye itself extremely small-too small to bring about per se a ręfractive change of 8.75 (No. 8) or 9 dioptres (Case II). A solution of sugar must be concentrated by 20 per cent. to bring about a change in its refractive power of 1 dioptre, and thus an increase of $1,000 \mathrm{gms}$. per dioptre would be necessitated.

(b) By this theory it is difficult to imagine why the refraction of every diabetic should not change. The phenomenon is comparatively rare. It would also necessitate a gradual rise and fall in refractive power, varying exactly with the sugar content; whereas the change, especially the hypermetropic change, is usually sudden.

(c) It cannot account for an astigmatic change, which occurs in 36 per cent. of cases in the series.

3. Affecting the lens. A lenticular change involving one or other, or both, of the following factors:

(i) A generalized change of mass and density, accompanied by a deformation of the anterior surface.

(ii) A change in the size and density of the cortical layer relative to the central nucleus (Grimsdale ${ }^{(2)}$ ), making the lens, for example in the hypermetropic change, a more uniform structure, tending to the infantile form, with a diminution of refractive power. 
There would appear to be at least six considerations pointing to the lens as being largely the causal agent in the phenomenon.

1. 81 per cent. of the cases in the series occurred between the ages of 40 and 60 years, the period of lenticular instability.

2. Elschnig(30) found that while one eye underwent changes, the other, on which a cataract extraction had been done, remained stationary. This is the only instance where one eye alone has been affected, and it would thus seem that the presence of the lens is necessary for the phenomenon to occur. lens."

3. Spalding ${ }^{(51)}$ has noticed "a shrinkage and flattening of the

4. The occurrence of astigmatic changes (in 36 per cent. of cases) suggests deformation of the lens as the origin.

5. It is conceivable to suppose that the character of this tissue and its mode of nourishment make it resistant to change; it is sluggish physiologically and pathologically. In the majority of diabetics changes do not occur. In some cases, after resisting osmotic forces for some time, it suddenly yields: this would account for what appears to be a very constant phenomenon and one somewhat difficult to explain. The sudden refractive change occurring some time after the sugar-value has fallen, while it also accounts for the rarity of the condition.

6. The lens in diabetes is unstable. The occurrence of cataract is common, and an opacity can readily be produced experimentally by sugar infusion in the frog ${ }^{(16)}$. Too close a parallelism should not, however, be drawn. It has been suggested that the myopia is comparable to that occurring prior to the sclerosing process of senile cataract, but this seems unlikely, in that the change is frequently sudden, there is a rapid improvement on treatment, lenticular opacities were detected in only 8.8 per cent. of the series, and such an analogy leaves no place for the incidence of an initial hypermetropia.

It seems most reasonable to explain these changes on the hypothesis that they are due to osmotic influences consequent on the disturbance of the water-balance of the body that forms so large a part in the general metabolic upset known clinically as diabetes. The conservation of normality in the constitution of the blood would appear to be one of the first and most urgent aims of the body-mechanism. A very small deviation from normal suffices to derange the nice equilibrium on which the processes of life depend: if there is any variation, the tendency is, at whatever cost, to keep it normal. As the blood-sugar increases the attempt is made, first, by the withdrawal, by osmosis, of fluid from the tissues, to dilute the sugar-laden blood, and secondly, to increase sugar excretion via the kidneys--(hence polyuria as a symptom). The net result of these two processes is to dehydrate the body, a 
catastrophe which is usually averted by a largely increased fluid intake-(hence polydipsia as a symptom). Pushed to extremes, however, the body is drained of its fluids, the eye shares in the general dehydration, by extraction of fluid its tension falls, the soft eye of diabetic coma results, and death usually supervenes. This has been demonstrated experimentally by Hertel (47) by sugar infusion in animals, and more recently corroborated in America ${ }^{(52)}$. Hertel found that such an eye contained on evaporation less fluid than normal; that the process was a purely physical one was shown in that the same phenomenon occurred on the infusion of neutral salts as chlorides, sulphates, phosphates, and carbonates; and that it was osmotical, in that the electrical conductivity of the eye was increased, demonstrating an increase in molecular concentration.

With a raised sugar we have thus a dilution of the blood by tissue fluids, and an increased excretion from the blood of a concentrated fluid with a high salt content, and a high osmotic pressure (urine), which is replaced volumetrically by drinking (practically) water. These together produce :

1. An increase in blood-volume,

2. A decrease in blood-salt content, both of which contribute to a lowering of osmotic pressure.

The change of volume may be explained thus: In health, the fluid traffic is regulated by the effective osmotic pressure of the blood over the lymph, due to the colloids of the former (20 to $30 \mathrm{~mm}$. Hg.), inducing a flow from the tissues to the blood stream, compensating for the amount lost by the filtration effected mechanically by the blood-pressure. In diabetes, the osmotic pressure is further raised by the presence of increased crystalloid (glucose); an effective increase over filtration pressure is thus established, and fluid in excess flows from the tissues to the blood, to be replaced, of course, by increased intake. The change in volume was compared in both cases (1) and (2) before and after changes in the sugar concentration were brought about, and the above reasoning was confirmed. The investigation was made by determinations of the red cell count and the haemoglobin percentage, for, since the number of the red cells does not change, and the fluid bulk is made up by a fluid lacking in haemoglobin, while these values remain constant absolutely, they undergo a percentage change relative to the volume. Thus after the exhibition of 50 gms., glucose to case (1), the count fell by about a million per c.mm. during the first hour (Fig. 4) and rose again as seen in the chart, and the haemoglobin fell from 90 per cent. to 70 per cent. As the blood-volume (other things being equal) varies inversely as the red cell count and the haemoglobin, it is seen that it varies directly with its sugar content.

To determine the effect of sugar variation on salt content, the total chlorides in the blood were estimated by Volhard's method 
before and after the occurrence of changes in cases 1 and 2 . Thus in case 1 , when the blood sugar was high on $25 / 3 / 24$, the salt content was 0.49 per cent. ; after the sudden fall it was 0.59 per cent. This lowering of the salt content in diabetes has been observed by others: Schmidt ${ }^{(53)}$, Meyers ${ }^{(54)}$, etc.; and MacLean ${ }^{(55)}$, estimating the chlorides in pathological conditions, found them lowest in diabetes, the decrease being due to a lowering of the normal chloride threshold. This is explained by the tendency for an excess in one crystalloid (glucose) to be compensated for by a depreciation in another (salt). That the salt-change will exert more osmotic disturbance, is evident from its smaller molecule and its ionization, since osmotic pressure varies as the number of molecules, and if these molecules are dissociated electrically, each fraction acts as an element equivalent osmotically to a molecule. It might be thought that the higher osmotic pressure established by glucose which causes the increase in volume, and the lower pressure entailed by decreased salt, were mutually antagonistic:, whereas they are complementary. Owing to its great diffusibility, salt equilibrium will be quickly established between the blood and tissue fluids. A fall in blood-salt thus demands a coincident fall in general fluidsalt. The larger sugar molecule diffuses more slowly, and therefore, while the salt equilibrium throughout is " set " at a lower level, the higher effective osmotic pressure in the blood due to excess crystalloid still is able relatively to effect changes in volume; thus on both counts, the osmotic pressure of the fluids bathing the lens is lowered.

Again another factor may enter into play. Applying the colloid chemical theory of absorption by protoplasm ${ }^{(56)}$, it is probable that, in the increased $\mathrm{PH}_{\mathrm{H}}$ of diabetic acidosis, with the subsequent buffering of the blood, some salt is transferred from an osmotically active state in the plasma to inactivity in the corpuscles, rendering the plasma still more dilute, and its osmotic pressure still less.

As the sugar content rises, therefore, the molecular concentration of the blood and tissue fluids falls. The lens, which we have already assumed to be peculiarly resistant to change, remains in statu quo, and is thus bathed by a fluid at a tension lower than its own molecular state; osmotic forces are set up, and to obtain equilibrium, fluid flows from the lower level to the higher, that is, into the lens, deforming it, and making the eye myopic. The increase of refractive power may be accentuated by a decrease in the index of refraction of the cortical layers, as opposed to the harder and more resistant nucleus, by dilution, thereby making the index of the whole lens less uniform, and therefore of a higher refractive power.

Conversely, on a fall of blood-sugar, a reverse osmotic change occurs, and some degree of hypermetropia is produced by the 
transference of fluid from the lens to the surrounding tissues and blood, whose osmotic pressure is now raised.

Cataract is common in diabetes. It would seem probable that its incidence may be partly accounted for by the ready degeneration of a lens previously weakened by the long continued strain of a deforming force, with the additional element of increased fluid traffic. Diabetes is a metabolic dyscrasia, notorious for the many complications it brings in its train-complications ranging in diversity and in severity from crops of boils to mass gangrene, from a peripheral neuritis, whose only clinical manifestation is a lost knee-jerk, to a widespread and profound paralysis. In the eye, the element of infection is seen in diabetic iritis : nerve involvement is exemplified in paralysis of accommodation, in retrobulbar neuritis, and in retinitis. The retina, like the lens, is nourished indirectly, and is, therefore, peculiarly susceptible to osmotic influence; and in nephritis, where again the water-and-salt balance is out of joint, the retina is affected.

Can it not be that these are all due to the same fundamental cause? The reaction between host-organism and invading microorganism is probably a question of the physico-chemistry of adsorption compounds, the excitation process of nerve, a matter of the diffusion of ions through semi-permeable membranes. It requires but a slight disturbance of osmotic forces to upset the nicely balanced equilibrium of the ionic and colloid systems on which the mechanism of life depends. At the least, reasoning thus, we approach more nearly the ideal concept, whereby the body, both in health and in disease, is an electro-chemical mechanism governed by purely physical laws, and get further removed from vitalistic views of normal and morbid processes, which are merely a dishonest confession of ignorance, are never the simplest, and are always sterile.

\section{Conclusions. I suggest that :}

1. In diabetes sudden changes of refraction occur. In the course of the disease with high or increasing sugar, myopia tends to occur : with decreasing sugar, and, therefore, usually just after the onset of energetic treatment, hypermetropia tends to occur.

2. The phenomenon is due to osmotic processes involving the lens, caused by a variation of the molecular concentration of the blood and tissue-fluids with the sugar content.

3. In both cases the tendency is to return to normal: in the first, under general diabetic treatment ; in the second, on the establishment of metabolic stability. Provided the diabetic element can be successfully dealt with, invariably a good prognosis can be give $n$ The patient can be assured that his eyes will return to norm a within a few weeks and that his vision will be unimpaired. 
4. In consequence of the general use of insulin, it is anticipated that the rarer condition of hypermetropia will become more common.

I am indebted to Dr. James Torrens for permission to publish the first case; to Dr. James Collier for the latter two; to Mr. R. R. James for his helpful and encouraging interest in the investigation; and to Mr. W. G. Laws for his kindness in supplying me with the notes of his two cases, and for his permission to add a note on them to this paper.

Since writing this paper, Mr. W. G. Laws has very kindly given me notes of two unpublished cases which have come under his observation where hypermetropia developed after the appearance of diabetes. Both patients were middle-aged women; the onset of hypermetropia was sudden, and occurred soon after the institution of treatment. In one the hypermetropia amounted to $4 \mathrm{D}$, and in the other to $2 \mathrm{D}$. In both the refraction returned to emmetropia when the diabetes was kept in check by treatment. In the first case a strict diet was instituted; the second case was given insulin, but not apparently in 1922 when the diabetes was first diagnosed and when the development of hypermetropia took place.

\section{BIBLIOGRAPHY OF CASES}

1. Appenzeller.-Centralbl. f. Augenheilk., p. 139, 1896.

2. Grimsdale. -Trans. Ophthal. Soc. U.K., No. 19, p. 267, 1899.

3. de Schweinitz.-Ophthal. Rec., p. 339, Dec., 1897.

4. Ibid, quoted by Gould. - Med. Rec., Vol. LXXI, No. 16, p. 633, 1907.

5. Dujardin. - Jl. des Scien. Méd. de Lille, July, 1900.

6. Heine.-Arch. f. Ophthal., No. 46, p. 541.

7 and 8. Neuberger.-Münch. Med. Wochenschr., No. 50, p. 506, 1903.

9. Alexander.-Klin. Monatsbl.f Augenheilk., No. 2, p. 108, 1903.

10. Landolt quoted by Culver. - "The Refraction and Accommodation of the Eye." 1904.

11. Jackson - Trans. Acad., Ophthal. and Otolaryn., p. 221, 1905.

12. Groenouw.-Augenleiden bei Diabetés mellitus, p. 46, 1907.

13. Wescott. - Trans. Sect. Ophtal., Amer. Med. Assoc. p. 296, 1911.

14 and 30.-Elschnig.-Med. Klin,, Berlin, No. 19, p. 17, 1923.

15. Hirschberg.-Deutsche Med. Wochenschr., No. 13, p 467, 1891.

16, 31, 32.-Risley.-Trans. Amer. Ophthal. Soc., No. 8, p. 121, 1897.

17. Gallus. -Zeits. f. Augenheilk., No. 15, p. 319. 1906.

18. Lundsgaard. - Zeits. f. Augenheilk., No. 17, p. 156, 1907.

19 and 36. Ibid.-Klin. Monatsbl. f. Augenheilk., No. 48, p. 38, 1910.

20 and 35. Knapp.-Zeits. f. Augenheilk., No. 21, p. 420, 1909.

21, 22, 38, 44. Ellis.-Trans. Sect. Obhthal. Amer. Med. Assoc., p. 296, 1911.

23. Zentmayer.-Annal. d'Ophtal., No. 21, p. 29, 1912.

24. Post.-Amer. Jl. of Ophthal., Nov., 1907.

25. Spalding quoted by Roberts. - Trans. Amer. Ophthal. Soc., No. 18, p. 408.

26 to $29,45 . \quad$ Roberts. - Trans, Amer. Ophthal. Soc., No. 19, p. 17, 1923.

33. Carpenter.-Ophthal. Rec., June, 1902.

34. Gould. -Med. Rec., No, 41, p. 633, 1907.

37. Lundsgaard. -Klin. Monatsbl. f. Augenheilk., No. 63, p. 356, 1919.

39. Horner.-Klin. Monatsbl. f. Augenheilk., p. 490, 1873.

40. Cohen.-Arch. f. Augenheilk. und Ohrenheilk., p. 38, 1878.

41. Doyne.-Trans. Ophthal. Soc. U.K., No, 19, p. 267, 1897.

42. Sourdille.-La Clin. Ophtal., No. 10, p. 125, 1900.

43. Lichtenstein.-Zeits. f. Augenheilk., No. 16, p. 330, 1906. 


\section{GENERAL REFERENCES}

46. Von Noorden.-Die Zuckerkrankheit, p. 127, 1912.

47. Hertel. - Mïnch. Med. Wochenschr., p. 1191, 1913.

48. Landolt and de Wecker. - Traité complet, iii., p. 388.

49. Keith, Rowntree and Geraghtry.-Arch. Intern. Med.. Chicago, No. 16, p. $547,1915$.

50. Fitz and Bock,-Jl. Biol. Chem., No, 48, p. 313, 1921.

51. Spalding, quoted by Joslin. - "Treatment of Diabetes Mellitus," Case No. 924, 1923.

52. Woodgate, Sansum and Wilder. Jl. Amer. Med. Assoc., No. 65, p. 2067, 1915 .

53. Schmidt.-Carakteristik der epidemische Cholera, Leipzig und Mitau, 1850.

54. Meyers.- " Practical Chemical Analysis of the Blood," p. 139, 1924.

55. MacLean.-Jl. Experim. Med., No. 22, pp. 212, 366, 1915.

56. Fischer.-Jl. Amer. Med. Assoc., No. 44, p, 325, 1915.

\section{ANNOTATIONS}

\section{The Visual Requirements of Motor Drivers}

In view of the fact that new legislation on the issue of motor drivers' licences is not unlikely in the immediate future it may be advisable to remind our readers that the Council of British Ophthalmologists issued a report in 1920 on the subject of visual requirements in motor drivers. This report, in which the whole ground is covered, was published in this journal (Vol. IV, 1920), and may still be obtained from the Council's secretary. The American Medical Association has recently published a report with recommendations read before its Ophthalmological Section (Jl. of Amer. Med. Assoc., Dec. 27, 1924) which does not confine itself to visual tests but deals with all physical defects in a manner that would certainly reduce the number of drivers and add to the incomes of the certifying physicians at the expense of those who were granted licences. Their recommendations are as follow:

"1. Every individual driving a motor vehicle, private, public or commercial, of the self-propelled type (automotive) shall be required to present to the motor licensing board in each state a certificate from a reputable physician (the standard of such medical fitness shall be licensure to practice medicine or surgery) in which the following points are certified :

(a) The applicant has no disqualifying defects of either legs or arms (anatomic or functional loss of hand, arm, foot or leg).

(b) The applicant has vision of at least $20 / 50$ in one eye and vision of at least 20/100 in the other eye with or without glasses.

(c) Double vision shall disqualify.

(d) All candidates must be able to hear a low spoken voice at 5 feet.

(e) The mentality of the applicant must be adequate and the heart's action reasonably healthy. 